\title{
THE PLACE OF ORIGIN OF THE FLICKERING IN Z CHA
}

\author{
ALBERT BRUCH \\ Astronomisches Institut \\ Wilhelm-Klemm-Str. 10 \\ D-48149 Münster, FRG
}

The variability in the observable light curve of a CV can be attributed to (i) orbital and long-term variations; (ii) flickering, (iii) photon noise, (iv) scintillation (negligible) and (v) any residual noise. Calculating the rmsscatter of the count rates in a light curve as a function of orbital phase, $\phi$, through accretion disk eclipse of high inclination CVs - after subtraction of a smoothed light curve to remove orbital and long-term variations, and applying a correction for photon noise - permits one to confine the place of origin of the flickering if its visibility is phase dependent or if eclipses take place (assuming any residual source of noise to be constant in time). The rms-scatter curve is then defined as the function $\sigma(\phi)=\sqrt{\sigma_{f}^{2}+\sigma_{r}^{2}}$, where $\sigma_{f}^{2}$ is the variance due to flickering and $\sigma_{r}^{2}$ the variance due to residual noise.

This method was applied to a large number of light curves of $\mathrm{Z}$ Cha in quiescence, normal maximum and super-maximum. The mean of the normalized rms-scatter curves of the individual light curves is shown in Fig. 1. The dashed and dotted vertical lines indicate the phases of the inner contact points of the white dwarf eclipse and the hot spot eclipse, respectively. Due to details of the subtraction of orbital and long-term variations the applied method is sensitive only to low-amplitude, small time-scale $(<1 \mathrm{~m})$ flickering. The phase dependence of the rms-scatter clearly indicates eclipses of the flickering light source. There are, however, significant differences depending on the photometric state of $\mathrm{Z}$ Cha.

Quiescence: The rms-scatter curve has a significant minimum centred on the total eclipse of the white dwarf. Furthermore, an enhanced scatter is evident during the phases of the orbital hump at $-0.26 \leq \phi \leq-0.02$, while it is somewhat lower between $0.02 \leq \phi \leq 0.3$, i.e. at phases when the hot spot is not visible. This suggest the presence of two different sources of flickering, one residing in the hot spot, the other close to the white dwarf. 


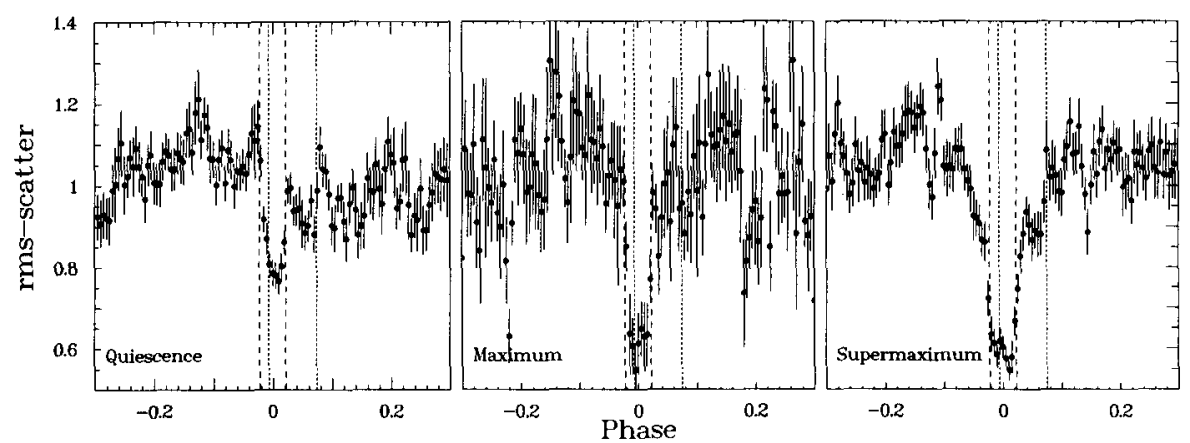

Figure 1. Rms-scatter curves of $\mathrm{Z}$ Cha in different photometric states

During the orbital hump phase both sources are visible, leading to enhanced scatter compared to the phases when the hot spot is invisible. When both white dwarf and hot spot are eclipsed the scatter assumes a minimum. A gradual decline of the rms-curve when the white dwarf - but not the hot spot - is totally eclipsed also argues for the contribution of hot spot flickering. A rapid increase of the scatter when the white dwarf emerges from the eclipse indicates that the white dwarf flickering is well localized in its immediate vicinity (the boundary layer?).

Maximum: In this case the rms-scatter curve attains a sharp, flatbottomed minimum during the phases of invisibility of the white dwarf, while at all other phases it remains on a level which - considering the strong noise - can be considered as constant. In particular, there is no significant difference before and after the eclipse. Thus, it can be concluded that the flickering is dominated by a contribution from the immediate vicinity of the white dwarf. Flickering of the hot spot, seen in quiescence, is completely outshone if it exists at all during outburst.

Super-maximum: Again the rms-scatter has a minimum centred on the eclipse phase of the white dwarf. However, in particular the egress of this minimum is more gradual, indicating the flickering light source to be more extended than during quiescence and normal outburst. Superposed is a broad asymmetrical feature with a gradual decrease of the rms-scatter in the interval $-0.07 \leq \phi \leq-0.03$ and a sudden increase (after a constant phase) close to $\phi=0.07$. Its broadness suggests that it is associated with an extended structure at the rim of the accretion disk. A more detailed analysis shows that the broad feature is present only when the superhump light source is not shining (superhump phase $0.25<\Phi<0.75$ ), while during superhump phases $-0.25<\Phi<0.25$, i.e. if the superhump maximum is roughly centred on orbital eclipse, the broad feature disappears and the ingress and egress of the eclipse of the flickering light source become significantly more gradual, indicating a large extension. 